\title{
HIGH-SPEED PHOTOMETRY OF CLUSTER FLARE STARS
}

\author{
M. RODONO \\ Catania Astrophysical Observatory, and Institute of Mathematics, University of Messina, Italy
}

\begin{abstract}
Recent photoelectric observations made at Catania Observatory show a high occurrence of short-lived faint flares on the Hyades member II Tau ( $\left.\mathrm{H}_{\text {II }} 2411\right)$. Its activity level turns out to be higher than for UV Cet-type stars of equal absolute luminosity.

II Tau could represent a transition prototype between UV Cet-type flare star in the solar neighbourhood and cluster ones.

The importance of coordinated photoelectric patrol of properly selected flare stars in clusters of different age is emphasized.
\end{abstract}

Photographic patrol observations of clusters of different ages, which have been carried out mainly at Asiago, Bjurakan, Konkoly and Tonantzintla Observatories, have shown the existence of a large number of flare stars and the general characteristics of their activity rates. The photographic patrol method has an appropriate ratio between information obtained and telescope time, being effective for problems related to (1) flare star discovery, (2) comparative flare frequency of stars in different clusters and, sometimes, (3) approximate knowledge of stellar flare parameters.

However, if we want to progress further in our knowledge of the flare phenomenon, more precise data on the activity of single objects in clusters of different ages are needed. To do this, photoelectric methods must be employed as for UV Cet-type stars in the solar neighborhood. Multi-colour photoelectric patrol of properly selected stars on each cluster should be made with time resolution as short as 1 to $3 \mathrm{~s}$.

The main difficulties are due to (1) the faintness of cluster flare stars, which requires medium-size to large aperture telescopes, (2) the natural reluctance of observers to engage in such time consuming researches and (3) the obvious unwillingness of telescope-time assignment committees to allocate a relatively large amount of telescope time to one field of research.

In a recent paper (Rodonò, 1974) we have shown how promising the photoelectric observations of cluster flare stars are. In this paper we present light curves with good time resolution of short-lived events on II Tau (HII 2411), which were obtained at McDonald Observatory with the $91-\mathrm{cm}$ and the $208-\mathrm{cm}$ Struve reflectors. These light curves have features in common with those of flares on UV Cet-type stars; for example, a relatively fast decline just after the maximum of light followed by a definitely slower decline or double peaked maximum.

Also, high-frequency oscillations have been detected during a rather intense flare observed at McDonald Observatory. The interpretation of this phenomenon needs, of course, additional observations both to confirm the reliability of the phenomenon itself and the study the physical triggering parameters.

More recent photoelectric observations of II Tau have been made at Catania Observatory with a $91-\mathrm{cm}$ cassegrain-type reflector, and a classical one-channel d.c. photometer with analogic output on a strip-chart recorder. The time response of the 
entire system is about $1 \mathrm{~s}$. During $9.15 \mathrm{~h}$ of effective patrol of the star, 14 flare events were observed. Their light curves are presented in Figure 1 and Figure 2 in the relative intensity scale $I_{\mathrm{f}} / I_{0}$ vs UT where $I_{\mathrm{f}}$ is the excess energy flux due only to the flare and $I_{0}$ the normal energy flux of the quiet star (outside flares). They are a rather faint and short-lived demonstrating proof of their relatively high frequency in agreement with the indications collected by Haro and Parsamian (1969) from the photographic observations. Moreover, some flares have complex light curves. So far, only Pigatto and Rosino (1971) have succeeded in detecting photographically the complex structure of a rather intense and prolonged event of the flare star Haro 41.

The mean flare frequency of $1.56 \mathrm{~h}^{-1}$ for II Tau observed in the fall of 1973 at Catania is significantly higher than that of $0.25 \mathrm{~h}^{-1}$ observed in the fall of 1972 at McDonald Observatory. However, the total energy emitted by the star through flares was higher in the latter period than in the former. A similar behaviour has been noticed for UV Cet (Cristaldi and Rodonò, 1969) and for other flare stars (Cristaldi

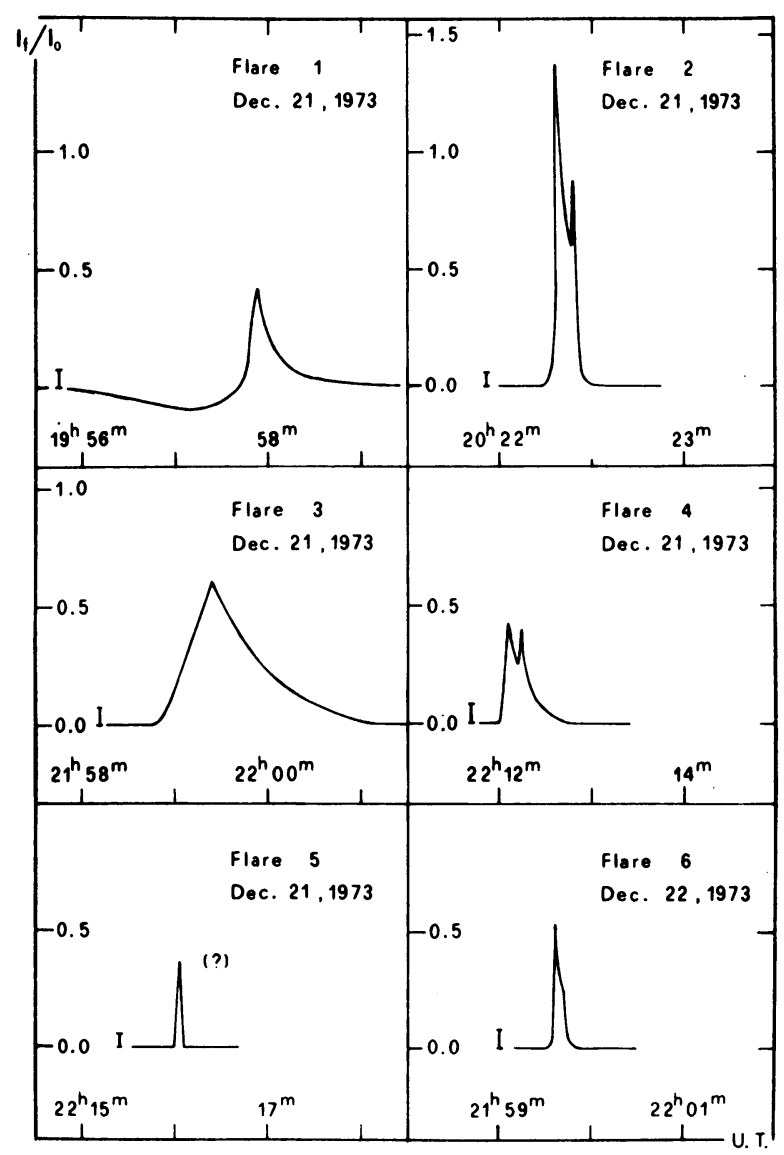

Fig. 1. Photoelectric light curves of II Tau (H II 2411) in the relative intensity scale vs UT. If represents the excess flux during flares with respect to the normal stellar flux $I_{0}$. 


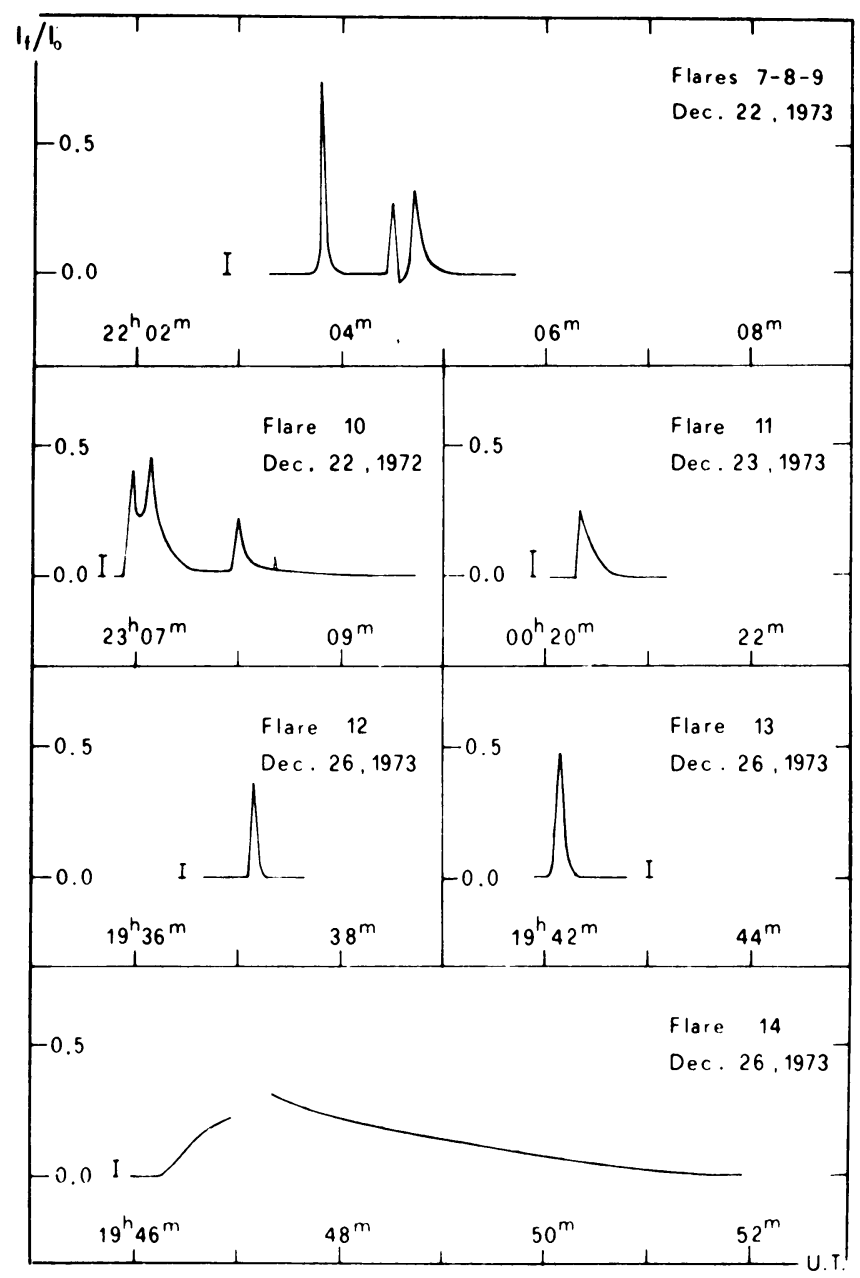

Fig. 2. Photoelectric light curves of II Tau ( $\mathrm{H}_{\text {II }}$ 2411) in the relative intensity scale vs UT. If represents the excess flux during flares with respect to the normal stellar flux $I_{0}$.

and Rodonò, unpublished). This indicates a modification of the flare activity with time, in that increasing the flare frequency leads to a lowering of the intensity of individual flares and of the total flare energy release.

A direct comparison of both the mean 'integrated intensity', i.e. the mean excess flux of the flare expressed in units of the flux per minute emitted by the quiet star, and the mean flare frequency of II Tau with respect to those of several UV Cet-type stars (Cristaldi and Rodonò, 1975) is presented in Figure 3. A B-mag. of 15.6 and distance modulus of 3.0 were adopted. Bearing in mind that the available photoelectric coverage of II Tau is shorter by at least one order of magnitude than that of the other flare stars included in the plot, no peculiar behaviour of the former can be 


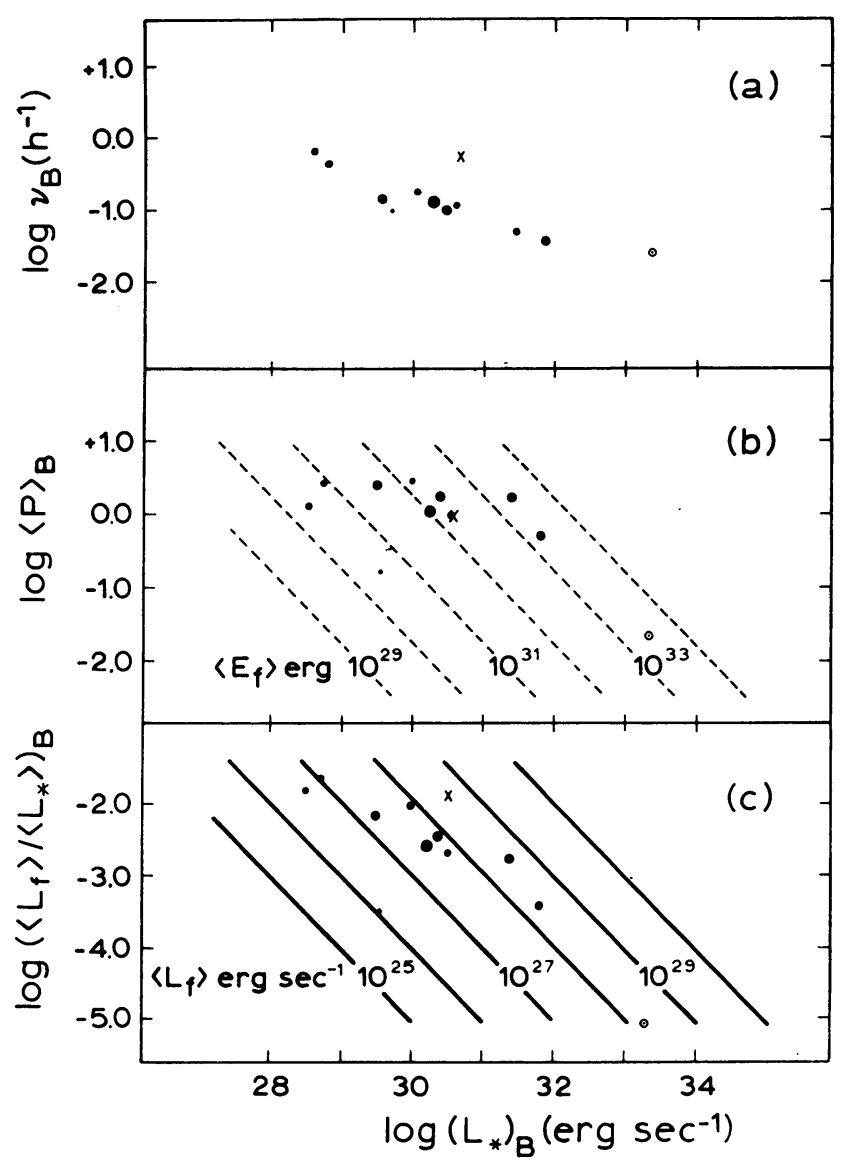

Fig. 3. Comparison of some flare activity parameters of II Tau $(\times)$ with those of UV Cet-type stars (•) vs $\log \left(L_{*}\right)_{B}$, the stellar luminosity in $B$-light: (a) mean flare frequency in $\mathrm{h}^{-1}$; (b) mean excess energy emitted during a flare in units of that emitted by the quiet star per minute, $\left\langle\boldsymbol{P}_{B}\right\rangle$; (c) average luminosity due to flare activity normalized to the star luminosity $\left\langle L_{\mathbf{f}}\right\rangle / L_{*}$. The two sets of parallel diagonals allow the absolute values of the parameters (b) and (c), referred to as $\left\langle E_{\mathfrak{l}}\right\rangle$ and

$\left\langle L_{\mathrm{p}}\right\rangle$, to be read. The data on UV Cet-type stars are taken from Cristaldi and Rodond (1974).

inferred. II Tau could represent a good transition prototype between UV Cet-type and cluster flare stars.

We believe that the preliminary results presented here are quite encouraging since they were obtained from only about $25 \mathrm{~h}$ of effective telescope time, mainly $91-\mathrm{cm}$ reflectors. But larger apertures are also needed to carry out simultaneous multi-colour photometry.

Investigating the basic problem of how flare activity changes with star age could require a large amount of photoelectric data. Therefore we should like to renew the recommendation which Ambartsumian and Mirzoyan (1971) made at the IAU Bamberg Colloq. No. 15 that people interested in this field should join their efforts 
under a coordinate programme of photoelectric observations of properly selected cluster flare stars.

\section{References}

Ambartsumian, V. A. and Mirzoyan, L. V.: 1971, IAU Colloq. 15, Veröff. Bamberg, No. 100, 98. Cristaldi, S. and Rodono, M.: 1969 IAU Inf. Bull. Var. Stars No. 404.

Cristaldi, S. and Rodond, M.: 1975, this volume, p. 75.

Haro, G. and Parsamian, E.: 1969 Bull. Obs. Tonantzintla Tacubaya 5, 41.

Pigatto, L. and Rosino, L.: 1971, IAU Colloq. 15, Veröff. Bamberg, No. 100, 116.

Rodond, M.: 1974, Astron. Astrophys. 32, 337.

\section{DISCUSSION}

L. A. Pustil'nik: What is the minimum resolution time?

M. Rodond: $1 \mathrm{~s}$.

L. A. Pustil'nik: What are the times of increase and decrease for the flare with rapid oscillations?

M. Rodonò: Increase about $1 \mathrm{~s}$; decrease about $100 \mathrm{~s}$.

L. Rosino: Is the flickering phenomenon as observed in HII 2411 during a flare periodic? And, if so, what was the period?

M. Rodono: The mean oscillation period is about $13 \mathrm{~s}$. 